\title{
Review of Heat Transfer Augmentation Through Different Passive Intensifier Methods
}

\author{
${ }^{1}$ Dr. ANIRUDH GUPTA, 2MAYANK UNIYAL \\ ${ }^{1}$ M.Tech, Phd (Iit Kanpur) Associate Professor Mechanical Department \\ ${ }^{2}$ M.Tech (Thermal) Mechanical Department B.T.K.I.T Dwarhat
}

\begin{abstract}
Heat transfer intensify techniques (passive, active or a combination of passive and active methods or compound methods) are commonly used in areas such as process industries, heating and cooling in evaporators, thermal power plants, air-conditioning equipment, refrigerators, radiators for space vehicles, automobiles, etc. Passive techniques, where inserts are used in the flow passage to intensify the heat transfer rate, are advantageous compared with active techniques, because the insert manufacturing process is simple and these techniques can be easily employed in an existing heat exchanger. In design of compact heat exchangers, passive techniques of heat transfer augmentation can play an important role if a proper passive insert configuration can be selected According to the heat exchanger working condition (both flow and heat transfer conditions). Many researchers have used different passive methods to improve heat transfer. Passive heat transfer methods are not only applicable in heat exchanger but also in solar air heater and cooling of electronic components (heat sink).
\end{abstract}

Keywords: Extended surfaces, heat exchangers, passive techniques, heat transfer enhancement, free and forced convection.

\section{Introduction}

Heat transfer inside flow passages can be enhanced by using passive surface modifications such as rib tabulators, protrusions, pin fins, and dimples. These heat transfer enhancement techniques have practical. Application for internal cooling of turbine airfoils, combustion chamber liners and electronics cooling devices, biomedical devices and heat exchangers.

The heat transfer can be increased by the following different Augmentation Techniques.

They are broadly classified into three different categories:

(i) Passive Techniques

(ii) Active Techniques

(iii) Compound Techniques.

\subsection{Passive techniques}

These techniques generally use surface or geometrical modifications to the flow channel by incorporating inserts or additional devices. They promote higher heat transfer coefficients by disturbing or altering the existing flow behavior (except for extended surfaces) which also leads to increase in the pressure drop.

In case of extended surfaces, effective heat transfer area on the side of the extended surface is increased. Passive techniques hold the advantage over the active techniques as they do not require any direct input of external power.

These techniques do not require any direct input of external power; rather they use it from the system itself which ultimately leads to an increase in fluid pressure drop. They generally use surface or geometrical modifications to the flow channel by incorporating inserts or additional devices. They promote higher heat transfer coefficients by disturbing or altering the existing flow behavior except for extended surfaces. Heat transfer augmentation by these techniques can be achieved by using;

(i) Treated Surfaces: Such surfaces have a fine scale alteration to their finish or coating which may be continuous or discontinuous. They are primarily used for Boiling and condensing duties.

(ii) Rough surfaces: These are the surface modifications that promote turbulence in the flow field in the wall region, primarily in single phase flows, without increase in heat transfer surface area.

(iii) Extended surfaces: They provide effective heat transfer enlargement. The newer developments have led to modified finned surfaces that also tend to improve the heat transfer coefficients by disturbing the flow field in addition to increasing the surface area.

(iv) Displaced enhancement devices: These are the inserts that are used primarily in confined forced convection, and they improve energy transport indirectly at the heat exchange surface by displacing the fluid from the heated or cooled surface of the duct with bulk fluid from the core flow. 
(v) Swirl flow devices: They produce and superimpose swirl flow or secondary recirculation on the axial flow in a channel. These include helical strip or cored screw type tube inserts, twisted tapes. They can be used for single phase and two-phase flows.

(vi) Coiled tubes: These lead to relatively more compact heat exchangers. It produces secondary flows and vortices which promote higher heat transfer coefficients in single phase flows as well as in most regions of boiling.

(vii) Surface tension devices: These consist of wicking or grooved surfaces, which direct and improve the flow of liquid to boiling surfaces and from condensing surfaces.

(viii) Additives for liquids: These include the addition of solid particles, soluble trace additives and gas bubbles in single phase flows and trace additives which usually depress the surface tension of the liquid for boiling systems.

(ix) Additives for gases: These include liquid droplets or solid particles, which are introduced in single- phase gas flows either as dilute phase (gas-solid suspensions) or as dense phase (fluidized beds).

\subsection{Active techniques}

These techniques are more complex from the use and design point of view as the method requires some external power input to cause the desired flow modification and improvement in the rate of heat transfer. It finds limited application because of the need of external power in many practical applications. In comparison to the passive techniques, these techniques have not shown much potential as it is difficult to provide external power input in many cases.

In these cases, external power is used to facilitate the desired flow modification and the concomitant improvement in the rate of heat transfer. Augmentation of heat transfer by this method can be achieved by:

(i) Mechanical Aids: Such instruments stir the fluid by mechanical means or by rotating the surface. These include rotating tube heat exchangers and scrapped surface heat and mass exchangers.

(ii) Surface vibration: They have been applied in single phase flows to obtain higher heat transfer coefficients.

(iii) Fluid vibration: These are primarily used in single phase flows and are considered to be perhaps the most practical type of vibration enhancement technique.

(iv) Electrostatic fields: It can be in the form of electric or magnetic fields or a combination of the two from dc or ac sources, which can be applied in heat exchange systems involving dielectric fluids. Depending on the application, it can also produce greater bulk mixing and induce forced convection or electromagnetic pumping to enhance heat transfer

(v) Injection: Such a technique is used in single phase flow and pertains to the method of injecting the same or a different fluid into the main bulk fluid either through a porous heat transfer interface or upstream of the heat transfer section.

(vi) Suction: It involves either vapor removal through a porous heated surface in nucleate or film boiling, or fluid withdrawal through a porous heated surface in single-phase flow.

(vii) Jet impingement: It involves the direction of heating or cooling fluid perpendicularly or obliquely to the heat transfer surface.

\subsection{Compound techniques}

A compound augmentation technique is the one where more than one of the above mentioned techniques is used in combination with the purpose of further improving the thermo-hydraulic performance of a heat exchanger.

When any two or more of these techniques are employed simultaneously to obtain enhancement in heat transfer that is greater than that produced by either of them when used individually, is termed as compound enhancement. This technique involves complex design and hence has limited applications.

\subsection{Mechanisms of Augmentation of Heat Transfer}

To the best knowledge of the authors, the mechanisms of heat transfer enhancement can be at least one of the following.

(1) Use of a secondary heat transfer surface.

(2) Disruption of the unenhanced fluid velocity.

(3) Disruption of the laminar sub layer in the turbulent boundary layer.

(4) Introducing secondary flows.

(5) Promoting boundary-layer separation.

(6) Promoting flow attachment/reattachment.

(7) Enhancing effective thermal conductivity of the fluid under static conditions

(8) Enhancing effective thermal conductivity of the fluid under dynamic Conditions

(9) Delaying the boundary layer development.

(10) Thermal dispersion. 
(11) Increasing the order of the fluid molecules.

(12) Redistribution of the flow.

(13) Modification of radiative property of the

(14) Increasing the difference between the surface and fluid temperatures.

convective medium.

(15) Increasing fluid flow rate passively.

(16) Increasing the thermal conductivity of the solid phase using special Nanotechnology fabrications

\section{Passive heat transfer techniques improved by the different researchers}

\section{Jian Yang Min Zeng Qiuwang Wang, Akira Nakayama [1]}

The forced convective heat transfer in three-dimensional porous pin fin channels is numerically studied in this paper. The Forchheimer-Brinkman extended Darcy model and two-equation energy model are adopted to describe the flow and heat transfer in porous media. Air and water are employed as the cold fluids and the effects of Reynolds number(Re), pore density (PPI) and pin fin form are studied in detail. The results show that, with proper selection of physical parameters, significant heat transfer enhancements and pressure drop reductions can be achieved simultaneously with porous pin fins and the overall heat transfer performances in porous pin fin channels are much better than those in traditional solid pin fin channels. The effects of pore density are significant. As PPI increases, the pressure drops and heat fluxes in porous pin fin channels increase while the overall heat transfer efficiencies decrease and the maximal overall heat transfer efficiencies are obtained at PPI_20 for both air and water cases. Furthermore, the effects of pin fin form are also remarkable. With the same physical parameters, the overall heat transfer efficiencies in the long elliptic porous pin fin channels are the highest while they are the lowest in the short elliptic porous pin fin channels.

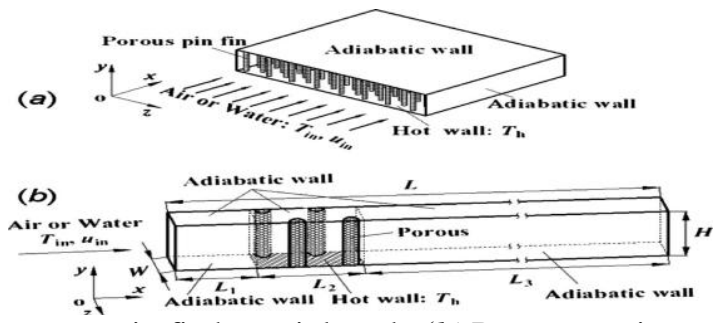

Physical model: „(a) porous pin fin heat sink and „(b) Representative computational domain
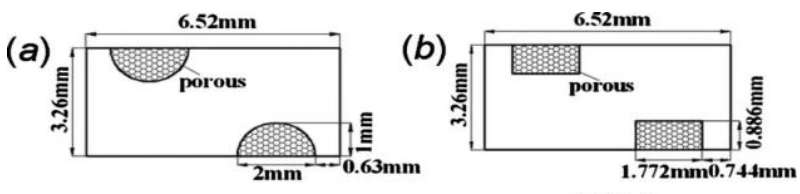

(c)

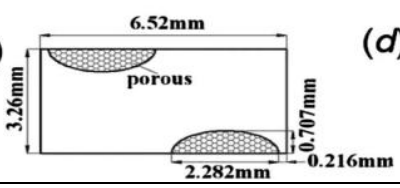

(d)

$6.52 \mathrm{~mm}$

Different forms of porous pin fin cross-section:

(a) circular Form $(b)$ cubic form $(c)$ long elliptic form, and $(d)$ short elliptic form

\section{Markus R"utten and Lars Krenkel}

The main physical mechanisms causing the enhancement of heat transfer is the generation and amplification of sufficiently strong longitudinal vortices which are interacting with the thermal boundary layer. The stratification of the thermal boundary layer near the heated walls is disturbed by these vortices. The convection of warmer fluid perpendicular to the heated wall and the mixing with colder fluid is intensified, and, additionally, further external momentum is transported into the inner boundary layer region.

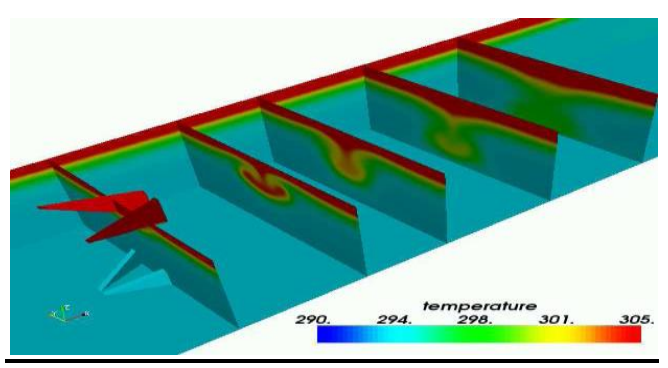

www.iosrjournals.org 


\section{K.S Dhanawade, H. S. Dhanawade}

The present paper reports, an experimental study to investigate the heat transfer enhancement in rectangular fin arrays with circular perforation equipped on horizontal flat surface in horizontal rectangular duct. The data used in performance analyses were obtained experimentally by varying flow, different heat inputs and geometrical conditions. The experiment covered Reynolds number range from 3000-6000, based on the flow average inlet velocity and hydraulic diameter. Clearance ratio $(\mathrm{C} / \mathrm{H}) 0.45$, inter-fin spacing ratio $(\mathrm{S} / \mathrm{H}) 0.22$, duct width $150 \mathrm{~mm}$, height $100 \mathrm{~mm}$ and fin size of both solid and perforated (weight reduction) were $100 \mathrm{~mm} \times 55 \mathrm{~mm}$ $\mathrm{x} 3 \mathrm{~mm}$. For various heat inputs and flow rates values of Reynolds and Nusselt number were obtained. The results of perforated fin arrays have been compared with its external dimensionally equivalent solid fin arrays. It shows that enhancement in heat transfer of perforated fin arrays than solid fin arrays.
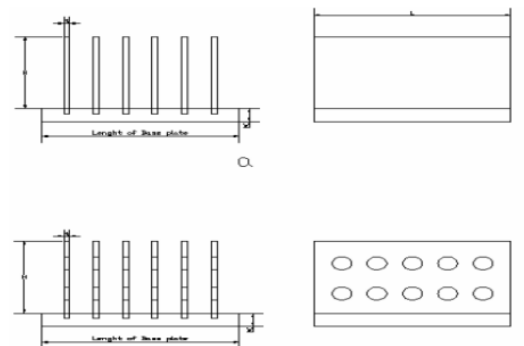

00000

Solid and perforated fin arrays

V.

M.J. Sable, S.J. Jagtap, P.S. Patil , P.R. Baviskar \& S.B. Barve

This heat transfer enhancing technique was investigated for natural convection adjacent to a vertical heated plate with a multiple v- type partition plates (fins) in ambient air surrounding. As compared to conventional vertical fins, this v-type partition plates works not only as extended surface but also as flow turbulator. In order to enhance the heat transfer, V-shaped partition plates (fins) with edges faced upstream were attached to the two identical vertical plates. The mica gladded nichrome flat heating element was sandwiched in between these two base plates. It is supplied with stabilized A.C.supply. The electrical heat input was controlled through dimmer stat and measured using a wattmeter. The V-type partition plates with two different heights were tried. The heat transfer in the downstream region of the partition plate is markedly enhanced when the plate height exceeds certain critical values because of the inflows of the low temperature fluid into the separation region. It was observed that among the three different fin array configurations on vertical heated plate, V-type fin array design performs better than rectangular vertical fin array and V-fin array with bottom spacing design. The performance was observed to improve further, with increase in the height of the V-plates (fin height).

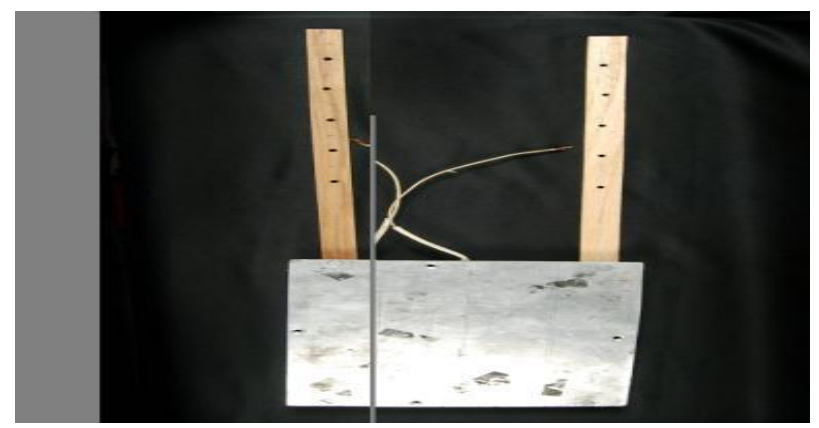

Plain vertical test plate $(250 \mathrm{~m} \times 250 \mathrm{~mm})$

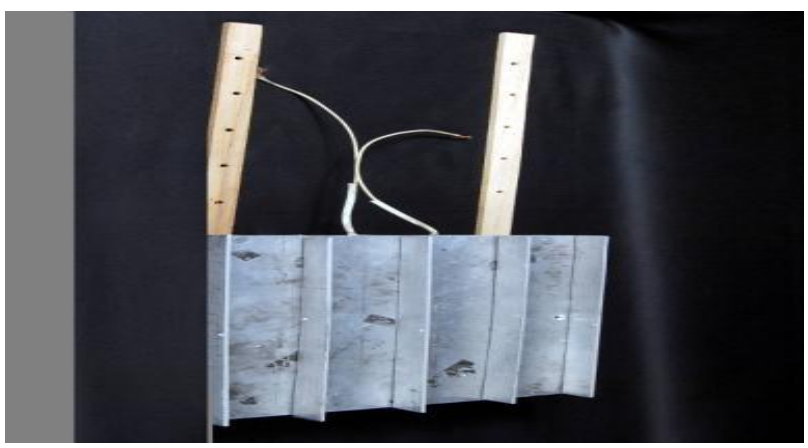

Vertical fin array (Fin height=20mm) 


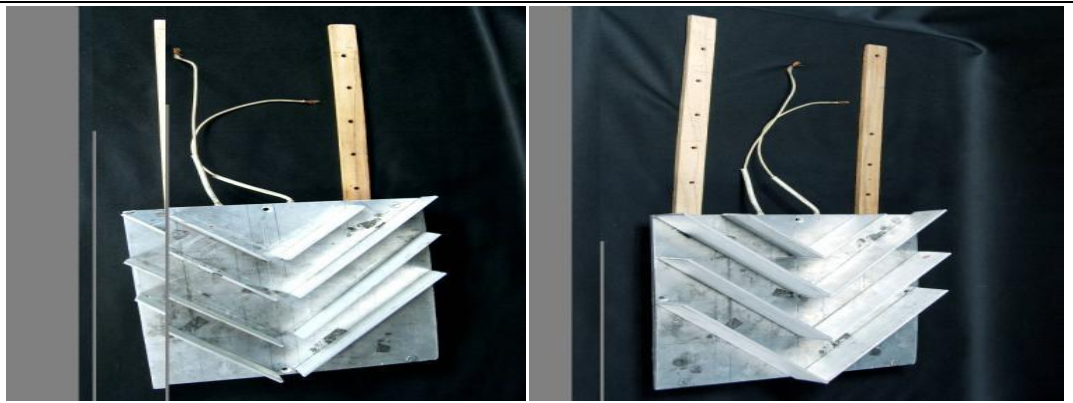

(V-fin array with bottom spacing $(20 \mathrm{~mm})$

\section{H. AlEssa and Q. Al-Odat [5]}

This study is aimed mainly at examining the extent of heat transfer enhancement from a horizontal rectangular fin under natural convection conditions as a result of introducing body modification (perforations) to the fin body. The modification in this work is vertical equilateral triangular perforations made through the fin thickness. The study investigates the influence of perforations on heat transfer rate or heat dissipation rate of the perforated fin. The modified fin (perforated fin) is compared to the corresponding solid (non-perforated) fin in terms of heat transfer rate. The study eventually attempts to make the best use of the material and size of a given fin, which involves some sort of optimization.

The specific objectives of the work may be summarized as follows:

1- Evaluate the influence of relevant fin and perforation factors on the enhancement of heat transfer rate.

2- Determine the values of parameters that would result in maximum heat transfer enhancement of the perforated fin compared to the solid counterpart.

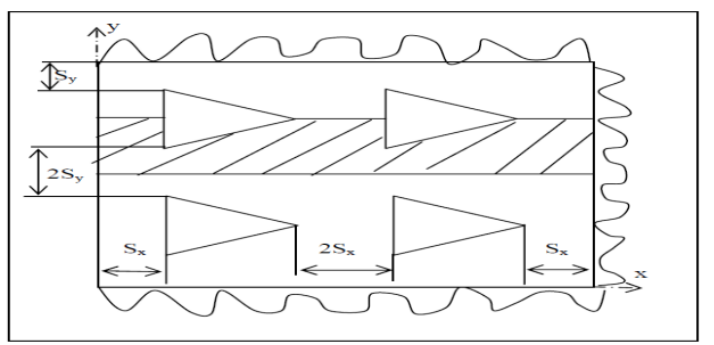

The symmetrical hatched part used in the mathematical formulation of the perforated fin

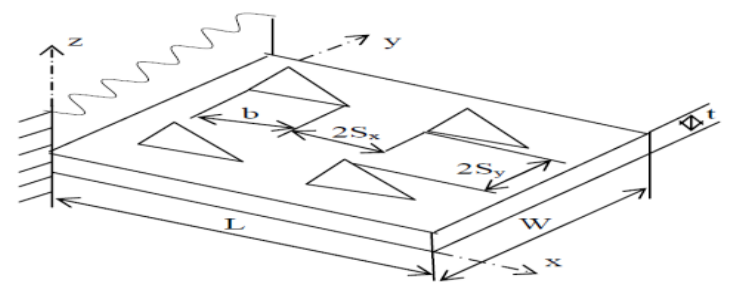

The fin with equilateral triangular perforations (perforation bases are parallel and toward the fin base)

\section{Yang, Wun, Youn Chen, Wang}

Pressure drop and heat transfer characteristics of different dimple vortex generators arrangement are examined in this study. A total of five heat sinks were made and tested, including plain fin, dimple, two-group dimple, oblique dimple gap 4-12 fin, oblique dimple gap6-12 fin. The tested results indicate that the pressure drop for dimple fin geometry is significantly higher than other fin types, followed bythe two group dimple fin. The fin structure with oblique dimple shows slightly increase of pressure drop as compared to the plain fin surface. The results show that more complicated fin structure will lead to higher pressure drop. The observed IR image of the temperature measurement of the test fin configurations also proves the measurements. The results suggest that the fin with dimple vortex generators is more beneficial than that of plain fin geometry. The oblique dimple fin is especially useful for air-cooling applicable for electronic devices to achieve effective augmentations without suffering from significant pressure penalty. 


\begin{tabular}{|c|c|c|}
\hline \multicolumn{3}{|c|}{ All tested heat sinks in the present investigation } \\
\hline Heat sink & Nomenclature & Side view \\
\hline (a) Plate & & \\
\hline (b) Dimple VG & & 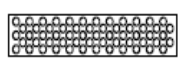 \\
\hline $\begin{array}{l}\text { (c) Two Groups } \\
\text { Dimple VG }\end{array}$ & & $\begin{array}{ll} \\
8\end{array}$ \\
\hline $\begin{array}{l}\text { (d) Oblique dimple } \\
\text { gap 4-12 fin }\end{array}$ & & 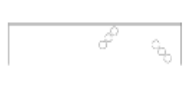 \\
\hline $\begin{array}{l}\text { (e) Oblique dimple } \\
\text { gap 6-12 fin }\end{array}$ & & 8 \\
\hline
\end{tabular}

VII. Kore, V. Joshi, K.Sane

An experimental investigation has been carried out to study heat transfer and friction coefficient by dimpled surface. The aspect ratio of rectangular channel is kept 4:1 and Reynolds number based on hydraulic diameter is varied from 10000 to 40000 . The ratios of dimple depth to dimple print diameter is varied from 0.02 to 0.04 to provide information on the influences of dimple depth. The ratio of channel height to print diameter is 0.5. The heat transfer and friction factor data obtained is compared with the data obtained from smooth plate under similar geometric and flow conditions. It is observed that at all Reynolds number as depth increases from 0.2 to 0.3 , the normalized Nusselt number and thermal performance increases and then after when depth increase from 0.3 to 0.4 normalized Nusselt number and thermal performance decreases. These are because of increase in strength and intensity of vortices and associated secondary flows ejected from the dimples.
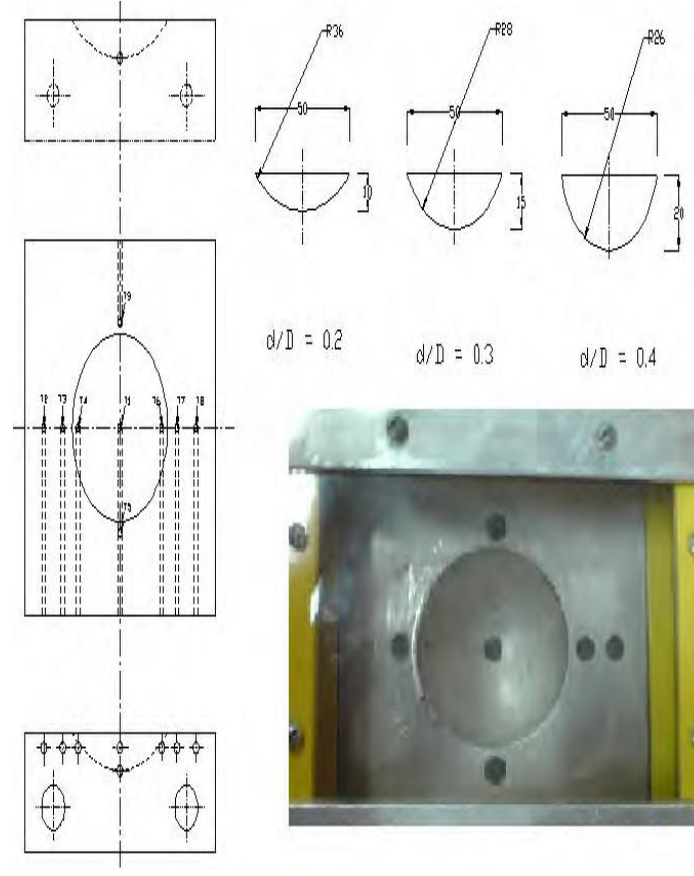

$d / D=0.2$

$d / D=0.3$

$d / D=0.4$

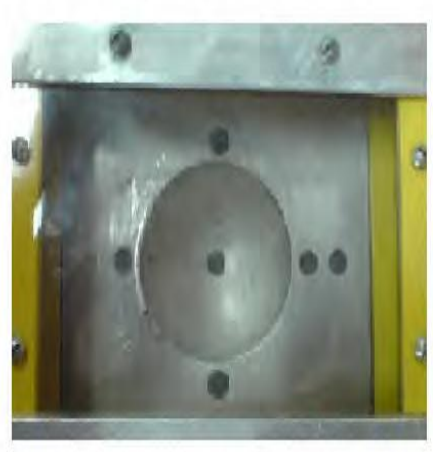

Dimple Geometry

\section{Wang., Yanxia, Zhang,}

The numerical analysis on convective heat transfer behavior and pressure drop characteristics of staggered circular tube bank fin heat exchanger with smooth fin and with interrupted half annular groove fin is carried out under the conditions of different tube rows $(1,2,3,4,5)$, different fin spans $(1.5 \mathrm{~mm}, 2 \mathrm{~mm}, 2.5 \mathrm{~mm})$ and different annular angles of interrupted half annular groove $\left(30^{\circ}, 50^{\circ}, 70^{\circ}\right)$, in addition, the velocity fields and the temperature fields of the structures are obtained. The results show that the ability of heat transfer could be enhanced by increasing tube rows, fin spans and annular angle of interrupted half annular groove in certain 
range, and the $\mathrm{Nu}$ number increases with the increase of Re number, whereas the resistance coefficient decreases with the increase of Re number in the heat exchangers both for the smooth fins and the interrupted half annular groove fins. The features of flow and heat transfer for heat exchanger with interrupted half annular groove fin are obtained by analysis of the formation, development and influence area of the vortices generated by interrupted half annular grooves.

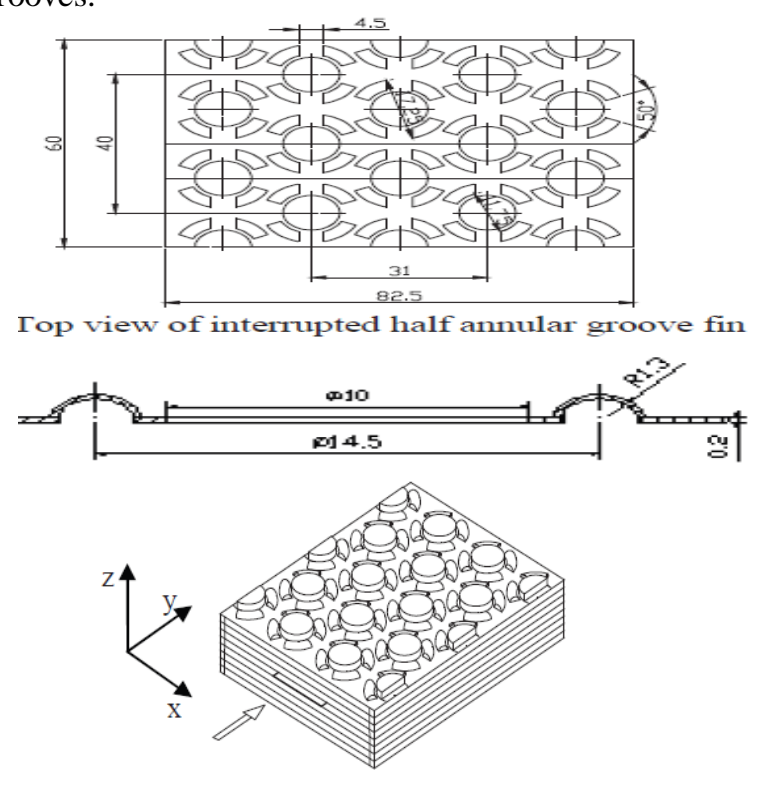

Sketch of heat exchanger with interrupted half annular groove fin

IX

S. V. Iyer and K. Vafai

In this work, free convective fluid flow and heat transfer are analyzed in a horizontal cylindrical annulus in the presence of a porous geometric perturbation. The flow in the porous region is modeled using the Brinkman-Forchheimer-Darcy model. The numerical scheme used in the present study is based on a Galerkin method of the finite element formulation. The nature of the three-dimensional flow field has been analyzed in detail, andthe local and average Nusselt numbers have been obtained for a range of Rayleigh numbers of practical interest. In order to evaluate the effect of a porous perturbation, the flow and heat transfer characteristics were compared with those for a regular annulus without a perturbation and one with a solid perturbation

\section{Vikrant Katekar B. Ankur VithalKar C. Bhojraj Kale}

Experimental investigation of thermal performance of solar air heater with roughened absorber plate. Solar air heater for high air velocity, flow becomes turbulent. Turbulent boundary layer with small laminar sublayer is formed on the absorber plate. Experimentally it has been found that laminar sublayer is formed on the absorber plate. Experimentally it has been found that laminar sub-layer offers very high resistance to heat flow. By breaking this layer or creating turbulent in this region, we can improve the heat transfer between absorber plate and air steam. Under the influence of artificial roughness on heat and flow characteristics, showed an important improvement in the thermal performance. The use of artificial roughness is found to be affective method to enhance the heat transfer coefficient.

\section{Seong-Yeon Yoo", Dong-Seong Park, Min-Ho Chung [11]}

Vortex generators are fabricated on the fin surface of a fin-tube heat exchanger to augment the convective heat transfer. In addition to horseshoe vortices formed naturally around the tube of the fin-tube heat exchanger, longitudinal vortices are artificially created on the fin surface by vortex generators. The purpose of this study is to investigate the local heat transfer phenomena in the fin-tube heat exchangers with and without vortex generators, and to evaluate the effect of vortices on the heat transfer enhancement.

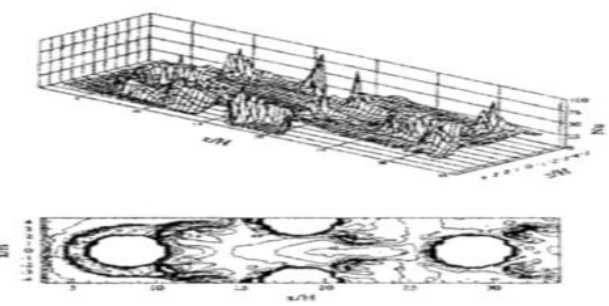

Distribution of local Nusselt number for fin circular tube heat exchanger with vortex generators 


\section{Conclusion}

Many researchers are taking interest to enhance heat transfer rate with passive methods.dimple, protrude and rough surfaces etc passive methods are used in heat exchangers, air heaters and heat sinks to enhance heat transfer. Passive methods can easily manufacture and applicable too. We need to work on compound technique, surly it will augment more heat transfer than other methods.

\section{References}

[1] Jian Yang Min Zeng Qiuwang Wang, Akira Nakayama "Forced Convection Heat Transfer Enhancement by Porous Pin Fins in Rectangular Channels" IEEE transactions on components and packaging technologies, vol. 27, no. 4, December 2004

[2] "Heat Transfer Enhancement by Using Vortex Generators" 5th European Congress on Computational Methods in Applied Sciences and Engineering (ECCOMAS 2008) June 30 -July 5, 2008 Venice, Italy

[3] K. H. Dhanawade, H. S. Dhanawade"Enhancement of Forced Convection Heat Transfer from Fin Arrays with Circular Perforation" IEEE 2010.

[4] M.J. Sable, S.J. Jagtap, P.S. Patil , P.R. Baviskar \& S.B. Barve"enhancement of natural convection heat transfer on vertical heated plate by multiple v-fin array" IJRRAS 5 (2) • November 2010.

[5] Abdullah H. AlEssa and Mohammed Q. Al-Odat "enhancement of natural convection heat transfer from a fin by triangular perforations of bases parallel and toward its base" The Arabian Journal for Science and Engineering, Volume 34, Number 2B, October 2009.

[6] Kai-Shing Yang, Kuo-Liang Wun, Ing Youn Chen, Chi-Chuan Wang "Experimental Investigation of High Performance Thermal Module with Dimple Vortex Generators" Department of Mechanical Engineering, National Chiao Tung University, Hsinchu Taiwan 300 .

[7] Sandeep S. Kore, Satishchandra V. Joshi, Narayan K.Sane "experimental investigations of heat transfer enhancement from dimpled surface in a channel" ISSN : 0975-5462 Vol. 3 No. 8 August 2011

[8] S. V. Iyer and K. Vafai, , "passive heat transfer augmentation in a cylindrical annulus utilizing a porous perturbation" 18 th Avenue, 1999,Columbus, OH 43210-1107,USA.

[10] A. Vikrant Katekar B. Ankur VithalKar C. Bhojraj Kale "enhancement of convective heat transfer coefficient in solar air heater of roughened absorber plate" Second International Conference on Emerging Trends in Engineering and Technology, ICETET-09

[11] Seong-Yeon Yoo", Dong-Seong Park, Min-Ho Chung, Sang-Yun Lee "Heat Transfer Enhancement for Fin-Tube Heat Exchanger Using Vortex Generators" KSME International Journal, VoL 16, No.1, pp. 109-115, 2002

[12] M. Siddique, A.-R. A. Khaled, N. I. Abdulhafiz, and A. Y. Boukhary International Journal of Chemical Engineering Volume 2010, Article ID 106461, 28 pages 